\title{
Metabolic Effects of Basal or Premixed Insulin Treatment in 5077 Insulin-Naïve Type 2 Diabetes Patients: Registry-Based Observational Study in Clinical Practice
}

\author{
Björn Eliasson • Nils Ekström • Sara Bruce Wirta • \\ Anders Odén $\cdot$ MirNabi Pirouzi Fard $\cdot$ Ann-Marie Svensson
}

To view enhanced content go to www.diabetestherapy-open.com

Received: March 20, 2014 / Published online: May 15, 2014

(c) The Author(s) 2014. This article is published with open access at Springerlink.com

\section{ABSTRACT}

Introduction: To investigate the clinical effects associated with premixed insulin (PM) and basal insulin [insulin NPH $(\mathrm{NPH})$, insulin glargine (IG), insulin detemir (ID)], in insulin-naïve patients with type 2 diabetes in routine clinical care.

Materials and Methods: Cohort study based on data from the Swedish National Diabetes Register, including 5,077 patients, resident in the Western region of Sweden. Patients were included between 1 July 2006 and 31 December

Electronic supplementary material The online version of this article (doi:10.1007/s13300-014-0068-9) contains supplementary material, which is available to authorized users.

B. Eliasson $(\bowtie) \cdot$ N. Ekström

Department of Medicine, University of Gothenburg,

Sahlgrenska University Hospital, 41345 Göteborg,

Sweden

e-mail: bjorn.eliasson@gu.se

S. Bruce Wirta

IMS Health, Stockholm, Sweden

\section{A. Odén}

Department of Mathematical Sciences, Chalmers

University of Technology, Göteborg, Sweden

M. P. Fard · A.-M. Svensson

Center of Registers in Region Västra Götaland,

Göteborg, Sweden
2009 and followed for 12 months. Changes in HbA1c, body mass index (BMI) and required insulin doses were compared between the different insulin types. Covariance adjustments were performed to adjust for differences between the groups.

Results: NPH, IG, ID and PM were all associated with significant reductions in HbA1c, mean \pm standard deviation ranged between $6.6 \pm 17.4 \mathrm{mmol} / \mathrm{mol} \quad$ (IG) and $8.9 \pm 17.7 \mathrm{mmol} / \mathrm{mol}(\mathrm{NPH})$, during the 12 months of follow-up. There were no statistically significant differences in the magnitude of $\mathrm{HbA1c}$ reduction between the insulin types. PM required 59\% higher and ID $25 \%$ higher insulin doses to achieve a similar HbA1c reduction as NPH. PM was associated with a significantly greater increase in BMI compared with NPH $(p=0.016)$, while IG and ID did not differ significantly from NPH. The number of patients experiencing severe hypoglycemia was low, but highest in patients treated with PM $(p=0.023)$.

Conclusions: NPH, IG, ID and PM were found to be equally effective in lowering $\mathrm{HbA1c}$ in insulin-naïve patients with type 2 diabetes in routine clinical care in Sweden. The effects on 
weight, dose and treatment persistence support the recommendation of NPH or IG as first and second choices in this group of patients requiring initiation of insulin treatment.

Keywords: Basal insulin; Glycosylated hemoglobin; Insulin detemir; Insulin glargine; Neutral protamine Hagedorn; Premixed insulin; Type 2 diabetes

\section{INTRODUCTION}

Current international guidelines promote the use of insulin treatment in patients with type 2 diabetes (T2D) when lifestyle changes and oral hypoglycemic agents (OHA) fail to achieve adequate glycemic control [1-3]. It is usually recommended to start with a basal insulin at bedtime, i.e., the medium long-acting neutral protamine Hagedorn (NPH) or a long-acting insulin analog [insulin glargine (IG) or insulin detemir (ID)], but premixed insulin (PM), usually administered twice daily, is a frequently used alternative [4]. IG and ID are advocated particularly in patients experiencing nocturnal hypoglycemia $[1,2]$, while the latter has been shown to require higher doses to achieve similar metabolic effects [5].

The clinical effects of these different insulin treatment regimens have been evaluated in randomized clinical trials (RCT) and subsequent meta-analyses, which together constitute the foundation of the treatment guidelines. Usually only RCTs are included in meta-analyses [6, 7]. Thus, the effects in clinical practice have generally not been described, although there are a small number of retrospective cohort studies that have evaluated different insulin therapies clinically [8-11]. One study compared different insulin-treatments in 4,337 insulin- naïve patients with T2D initiating on $\mathrm{NPH}$, PM, IG, or ID in routine clinical practice, using a retrospective database approach [The Health Improvement Network (THIN) database] [12]. Glycosylated hemoglobin (HbA1c), weight gain and insulin dose were evaluated during 12 months and persistence during 36 months, showing that patients initiating on NPH experienced a modest disadvantage in glycemic control, IG achieved best HbA1c reduction, while PM showed greatest weight gain and highest insulin dose, but showed superior persistence.

The authors initiated this register-based project to study the effects of four insulin regimens in 5,077 insulin-naïve patients with T2D, resident in the geographical region of Western Sweden (Region Västra Götaland), the clinical effects, such as changes in HbA1c and body mass index (BMI), insulin dose, and frequencies of hypoglycemia. Another aim of the project was to investigate health care utilization and costs, but these results will be presented in a subsequent report.

\section{METHODS}

This is a retrospective cohort study based on information retrieved from four national health registers: the Swedish National Diabetes Register (NDR), the Prescribed Drug Register, the Cause of Death Register and the Regional Claims Database (VEGA) of the Region Västra Götaland. All procedures followed were in accordance with the ethics review board at the University of Gothenburg and with the Helsinki Declaration of 1975, as revised in 2000 and 2008. All included patients have agreed by informed consent to be registered before inclusion. 


\section{Databases}

NDR was initiated in 1996 to enable local quality control and regional benchmarking against national treatment guidelines [13]. Trained physicians and nurses via the Internet or clinical record databases carry out annual reporting to the NDR during patient visits at hospitals and primary health care centers nationwide. Thus, clinical data such as BMI, HbA1c, and debut of diabetes were collected from NDR. The Prescribed Drug Register (PDR) contains full coverage of all filled drug prescriptions (pharmaceutical agents and amounts) at pharmacies in Sweden [14]. The Cause of Death Register contains information about causes of mortality and death dates [15] and the VEGA database comprises information about diagnoses [International Classification of Diseases (ICD)-10 and Diagnosis Related Groups (DRG) codes], performed procedures [Nordic Medico-Statistical Committee (NOMESCO) and local procedure codes] and hospital length of stay for inpatient, outpatient, primary, and private care for all inhabitants in the Region Västra Götaland.

\section{Inclusion and Exclusion Criteria}

Insulin-naïve patients with $\mathrm{T} 2 \mathrm{D}$ resident in the Region of Västra Götaland, who were at least 18 years old and initiated continuous use of basal insulin (NPH, IG, ID) or PM, were included (Fig. 1). The definition of T2D was treatment with diet only, OHA only, or onset age of diabetes $\geq 40$ years and treatment with insulin only or combined with OHA. Insulin-naïve patients were identified by exclusion of patients who had a prescription of any type of insulin filled between 1 July 2005 and 30 June 2006.

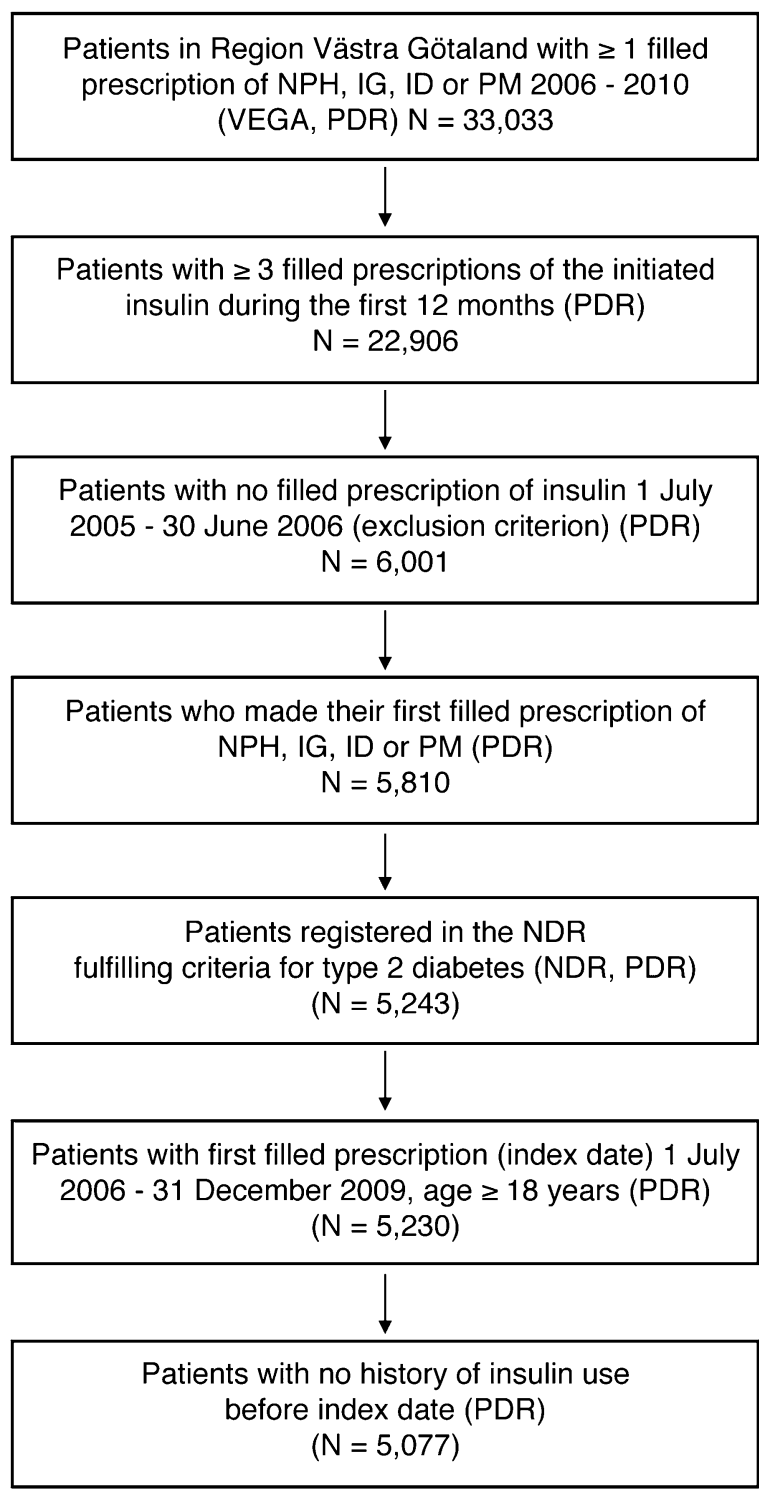

Fig. 1 Patient disposition. Stages of inclusion and exclusion of patients, and used databases. $I D$ insulin detemir, $I G$ insulin glargine, NDR National Diabetes Register, NPH neutral protamine Hagedorn, $P M$ premixed insulin, $P D R$ prescribed drug register, VEGA Regional Claims Database of the Region Västra Götaland

\section{Study Period, Follow-up and Censoring}

The study period was between 1 July 2005 and 31 December 2010. Patients were required to have their first prescription of insulin filled (index date) between 1 July 2006 and 31 
December 2009 to allow for 1 year of follow-up. Patients were thus followed for 12 months or until the occurrence of a censoring event. Start of follow-up was defined as the date of the first filled insulin prescription (index date) in each patient. Censoring events included a filled prescription of a new type of insulin (NPH, IG, ID or PM), death or move out of the Region Västra Götaland.

\section{Patient Characteristics}

Variables measured at baseline (index date) included: age, gender, level of income, diabetes duration, previous OHA usage, a history of diabetes complications, or cardiovascular disease (CVD). The latest HbA1c- and BMI-values up to 6 months before index date were used for this. To calculate the levels of these variables at the end of the followup period, the authors used the values closest, i.e., values within a period ranging 3 months prior to and 3 months following end of followup. History of CVD was defined as diagnosis of ischemic heart disease (I20-I25), atrial fibrillation (AF) (I48), congestive heart failure (CHF) (I50), or stroke (I61, I63, I64, I67.9) prior to the index date. History of diabetes complications was defined as diagnosis of diabetic ulcer (E11.62), neuropathy (E11.4), nephropathy (E11.2) or microalbuminuria (urine albumin excretion rate $>20 \mu \mathrm{g} / \mathrm{min}$ in two of three consecutive tests) prior to the index date. History of OHA usage was defined as at least one filled prescription of any OHA between 1 July 2005 and index date. Furthermore, the number of different OHAs prescribed during this period was taken into account. Median income for different municipalities of residence were retrieved from Statistics Sweden, and divided into quartiles ranging from high to low income.

\section{Outcomes}

This investigation studied changes in HbA1c and in BMI and number of hypoglycemic events. A hypoglycemic event was defined as ICD-10-codes E10.0C, E11.0C, E16.0, E16.1, E16.1W and E16.2 to capture all possible cases. Furthermore, mean daily insulin doses were calculated in each group as the total prescribed dose during follow-up divided by the number of days between index date and end of follow-up. Mean insulin doses per day and kilogram were calculated for patients with available information on weight. Analyses of HbA1c were carried out at local laboratories. During the study period, HbA1c analyses were quality assured nationwide by regular calibration with the high-performance liquid chromatography Mono-S method.

\section{Statistical Methods}

Baseline characteristics are presented as means \pm 1 standard deviation (SD) or medians for continuous variables and frequencies for categorical variables with crude significance levels for differences between the groups, when analyzed using ANOVA or $\chi^{2}$ test (Table 1). For continuous variables with nonnormal distribution, a Kruskal-Wallis test was performed. All continuous outcome variables were explored for their distribution. A correlation analysis was performed between pre-index and post-index values for $\mathrm{HbA1c}$ and BMI, in order to determine the strength of the correlation and the appropriate means of analysis. If the correlation coefficient exceeded 0.5 , the incremental value (change from pre- to post-index) was used in the regression, while the pre-index value was included as a covariate in the regression in cases with lower correlation coefficients. 
Table 1 Clinical characteristics

\begin{tabular}{lllllc}
\hline & $\begin{array}{l}\text { NPH } \\
(\boldsymbol{n}=\mathbf{2 , 4 9 0})\end{array}$ & IG $(\boldsymbol{n}=\mathbf{6 8 0})$ & ID $(\boldsymbol{n}=\mathbf{1 5 8})$ & $\begin{array}{l}\text { PM } \\
(\boldsymbol{n}=\mathbf{1 , 7 4 9 )})\end{array}$ & $\boldsymbol{p}$ value \\
\hline Male gender (\%) & 61 & 59 & 56 & 55 & 0.0017 \\
Age [years (mean \pm SD)] & $65 \pm 13$ & $64 \pm 14$ & $59 \pm 14$ & $71 \pm 13$ & $<0.001$ \\
Diabetes duration [years (mean \pm SD)] & $7.5 \pm 6.0$ & $8.6 \pm 7.1$ & $7.6 \pm 5.5$ & $8.4 \pm 6.5$ & $<0.001$ \\
History of CVD (\%) & 34 & 32 & 22 & 44 & $<0.001$ \\
History of diabetes complications (\%) & 47 & 46 & 32 & 50 & $<0.001$ \\
OHA use (\%) & 77 & 80 & 84 & 77 & 0.065 \\
Number of OHA (mean \pm SD) & $1.4 \pm 1.0$ & $1.6 \pm 1.1$ & $1.6 \pm 1.1$ & $1.4 \pm 1.1$ & $<0.001$ \\
\hline
\end{tabular}

$C V D$ cardiovascular disease, $I D$ insulin detemir, $I G$ insulin glargine, $N P H$ neutral protamine Hagedorn, $O H A$ oral hypoglycemic agent, $P M$ premixed insulin, $S D$ standard deviation

The authors performed multivariate analysis of the outcomes post-index $\mathrm{HbA} 1 \mathrm{c}(\mathrm{mmol} / \mathrm{mol})$, incremental BMI $\left(\mathrm{kg} / \mathrm{m}^{2}\right)$ and daily insulin dose (units/day kg), using generalized linear modeling. For each outcome, three models were explored and presented: firstly, unadjusted where outcome was as a function of insulin groups; secondly, including covariates with few missing values (age, gender, level of income, diabetes duration, history of CVD, history of diabetes complications, previous OHA use, and followup time); and finally, a fully adjusted model including age, gender, level of income, diabetes duration, history of CVD, history of diabetes complications, previous OHA use, follow-up time, pre-index HbA1c, pre-index BMI and weight. Some covariates were not considered appropriate to include for all outcomes. Followup time was only included as a covariate when evaluating HbA1c, and BMI, while for evaluating daily insulin dose and censoring, follow-up time was either intrinsic to the outcome or used for estimating the outcome. For the same reason, weight was only included for HbA1c and censoring.
All statistical analyses were performed in SAS V.9.3 (SAS Institute, Cary, North Carolina, USA). A two-sided $p$ value $<0.05$ was considered statistically significant.

\section{RESULTS}

In total, 5,077 insulin-naïve patients with T2D were included in the study. The majority were initiated on NPH (49\%) or PM (34\%), while 13\% and $3 \%$ were initiated on IG and ID, respectively. There were overall significant differences in clinical characteristics between the groups (Table 1), but we did not compare the different groups separately. Those initiated on ID were numerically youngest with the lowest proportions of CVD and diabetes complications, while patients receiving PM were the oldest and had the highest proportions of CVD and diabetes complications. Patients initiated on NPH and IG were quite similar in clinical characteristics, with about $60 \%$ males, a mean age of about 65 years, a history of CVD in approximately one-third and diabetes complications in about half of the patients, but with a longer diabetes 
Table 2 Length of follow-up and censoring

\begin{tabular}{llllll}
\hline & NPH & IG & ID & PM & $\boldsymbol{p}$ value \\
\hline Mean follow-up [days (mean \pm SD)] & $352 \pm 47$ & $358 \pm 37$ & $351 \pm 51$ & $352 \pm 47$ & 0.0099 \\
Any censoring $(n, \%)$ & $244,9.8$ & $39,5.7$ & $16,10.1$ & $165,9.4$ & 0.023 \\
Censored due to death $(n, \%)$ & $60,2.4$ & $16,2.4$ & $1,0.6$ & $78,4.5$ & $<0.001$ \\
Censored due to switch $(n, \%)$ & $173,6.9$ & $21,3.1$ & $13,8.2$ & $81,4.6$ & $<0.001$ \\
Censored due to move $(n, \%)$ & $11,0.4$ & $2,0.3$ & $2,1.3$ & $6,0.3$ & NS \\
\hline
\end{tabular}

$I D$ insulin detemir, $I G$ insulin glargine, $N P H$ neutral protamine Hagedorn, $N S$ not significant, $O H A$ oral hypoglycemic agent, $P M$ premixed insulin, $S D$ standard deviation

duration in the IG group $(8.6 \pm 7.1$ years compared to $7.5 \pm 6.0$ years).

Differences in mean follow-up time between the groups were small but statistically significant, with follow-up time ranging from $351 \pm 51$ days in ID to $358 \pm 37$ days in IG (Table 2). The proportion of patients censored due to any cause was lowest in IG, mostly due to less treatment switches (3.1\%) compared to $\mathrm{NPH}$ (6.9\%), ID (8.2\%) and PM (4.6\%). There were significant differences in censoring due to death, with the highest proportion seen in PM $(4.5 \%)$ and the lowest proportion seen in ID $(0.6 \%)$. However, this analysis was not adjusted for differences in clinical characteristics between the groups.

Table 3 describes mean \pm SD for HbA1c and BMI at baseline, end of follow-up, as well as unadjusted changes in HbA1c and BMI during follow-up in the different groups. HbA1c levels at baseline ranged between $67 \pm 16 \mathrm{mmol} / \mathrm{mol}$ (PM) and $68 \pm 17 \mathrm{mmol} / \mathrm{mol}(\mathrm{NPH})$ with no statistically significant differences between the groups. Change in HbA1c also did not differ significantly between the groups, ranging between $-7 \pm 17 \mathrm{mmol} / \mathrm{mol}$ (IG) and $-9 \pm 18$ $\mathrm{mmol} / \mathrm{mol}$ (NPH). The non-significant differences in change of HbA1c remained when IG, ID or PM was compared with NPH as reference, adjusted for covariates. There were small differences in BMI between the groups, with patients treated with ID being most obese $\left(32.1 \pm 7.1 \mathrm{~kg} / \mathrm{m}^{2}\right)$ and patients treated with PM being least obese $\left(29.5 \pm 5.4 \mathrm{~kg} / \mathrm{m}^{2}\right)$ at baseline. This pattern remained during the study even though patients starting PM gained most weight, with a change in BMI of $0.8 \pm 2.3$ $\mathrm{kg} / \mathrm{m}^{2}$ compared to $0.4 \pm 1.8,0.3 \pm 2.3$ and $0.3 \pm 2.3 \mathrm{~kg} / \mathrm{m}^{2}$ in $\mathrm{NPH}, \quad \mathrm{IG}$ and ID, respectively. When BMI change was compared in IG, ID and PM with NPH as reference, adjusted for covariates, the increase in BMI remained significantly greater in PM, $p=0.016$ (Tables 4, 5, 6).

There were significant differences in insulin doses between the groups (Table 7). Among patients receiving insulin in monotherapy, the weight-adjusted doses were highest in PM and ID. However, when doses of mealtime insulins were taken into account, only ID required higher doses. In patients treated with insulin in combination with OHA, both ID and PM required higher weight-adjusted doses even when mealtime insulin use was taken into account. NPH and IG required similar weightadjusted doses in patients receiving insulin in monotherapy as well as in patients with insulin in combination with OHA. Covariance-adjusted comparisons of weight-adjusted daily insulin doses with IG, ID or PM and NPH as reference 
Table 3 Baseline values and changes in HbAlc and BMI

\begin{tabular}{lccccc}
\hline & NPH $($ mean \pm SD) & IG (mean \pm SD) & ID (mean \pm SD) & PM (mean \pm SD) & $p$ value \\
\hline HbAlc pre-index $(\mathrm{mmol} / \mathrm{mol})$ & $68 \pm 17$ & $67 \pm 17$ & $68 \pm 17$ & $67 \pm 16$ & $\mathrm{NS}$ \\
HbAlc post-index $(\mathrm{mmol} / \mathrm{mol})$ & $59 \pm 12$ & $61 \pm 14$ & $61 \pm 16$ & $59 \pm 12$ & $\mathrm{NS}$ \\
HbAlc change $(\mathrm{mmol} / \mathrm{mol})$ & $-9 \pm 18$ & $-7 \pm 17$ & $-7 \pm 18$ & $-8 \pm 17$ & $\mathrm{NS}$ \\
BMI pre-index $\left(\mathrm{kg} / \mathrm{m}^{2}\right)$ & $30.9 \pm 5.4$ & $30.2 \pm 5.4$ & $32.1 \pm 7.1$ & $29.5 \pm 5.4$ & 0.0022 \\
BMI post-index $\left(\mathrm{kg} / \mathrm{m}^{2}\right)$ & $31.3 \pm 5.4$ & $30.5 \pm 5.6$ & $32.4 \pm 7.2$ & $30.3 \pm 5.8$ & 0.033 \\
BMI change $\left(\mathrm{kg} / \mathrm{m}^{2}\right)$ & $0.4 \pm 1.8$ & $0.3 \pm 2.3$ & $0.3 \pm 2.3$ & $0.8 \pm 2.3$ & 0.048 \\
\hline
\end{tabular}

$B M I$ body mass index, $H b A l c$ glycosylated hemoglobin, $I D$ insulin detemir, $I G$ insulin glargine, $N P H$ neutral protamine Hagedorn, $N S$ not significant, $P M$ premixed insulin, $S D$ standard deviation

Table 4 Adjusted comparison of post-index HbAlc in IG, ID, PM vs. NPH

\begin{tabular}{|c|c|c|c|c|c|c|c|c|c|}
\hline \multirow{2}{*}{$\begin{array}{l}\text { Post-index HbAlc } \\
(\mathrm{mmol} / \mathrm{mol})\end{array}$} & \multicolumn{3}{|c|}{ Model 1, $n=2,534$} & \multicolumn{3}{|c|}{ Model 2, $n=993$} & \multicolumn{3}{|c|}{ Model 3, $n=778$} \\
\hline & $\overline{\text { Change }}$ & SD & $p$ value & Change & SD & $p$ value & Change & SD & $p$ value \\
\hline IG vs. NPH & 1.0 & 0.01 & 0.005 & 1.0 & 0.02 & 0.092 & 1.0 & 0.02 & 0.44 \\
\hline ID vs. $\mathrm{NPH}$ & 1.1 & 0.02 & 0.000 & 1.0 & 0.04 & 0.57 & 1.0 & 0.04 & 0.43 \\
\hline PM vs. NPH & 1.0 & 0.01 & 0.44 & 1.0 & 0.01 & 0.56 & 1.0 & 0.02 & 0.59 \\
\hline
\end{tabular}

Multivariate analysis using generalized linear modeling. Model 1, unadjusted; Model 2, adjusted for covariates with few missing values (age, gender, level of income, diabetes duration, history of CVD, history of diabetes complications, previous OHA use, and follow-up time); Model 3, fully adjusted as Model 2 plus pre-index HbAlc, pre-index BMI and weight $B M I$ body mass index, $H b A 1 c$ glycosylated hemoglobin, $I D$ insulin detemir, $I G$ insulin glargine, $N P H$ neutral protamine Hagedorn, $O H A$ oral hypoglycemic agent, $P M$ premixed insulin, $S D$ standard deviation

Table 5 Adjusted comparison of BMI change of IG, ID, PM vs. NPH

\begin{tabular}{|c|c|c|c|c|c|c|c|c|c|}
\hline \multirow{2}{*}{$\begin{array}{l}\text { Incremental BMI } \\
\left(\mathrm{kg} / \mathrm{m}^{2}\right)\end{array}$} & \multicolumn{3}{|c|}{ Model $1(n=924)$} & \multicolumn{3}{|c|}{ Model $2(n=868)$} & \multicolumn{3}{|c|}{ Model $3(n=759)$} \\
\hline & Change & SD & $p$ value & Change & SD & $p$ value & Change & SD & $p$ value \\
\hline IG vs. NPH & -0.2 & 0.2 & 0.46 & -0.2 & 0.2 & 0.40 & -0.2 & 0.2 & 0.42 \\
\hline ID vs. NPH & -0.1 & 0.4 & 0.77 & -0.2 & 0.4 & 0.57 & -0.3 & 0.4 & 0.44 \\
\hline PM vs. NPH & 0.4 & 0.2 & 0.017 & 0.4 & 0.2 & 0.009 & 0.4 & 0.2 & 0.016 \\
\hline
\end{tabular}

Multivariate analysis using generalized linear modeling. Model 1, unadjusted; Model 2, adjusted for covariates with few missing values (age, gender, level of income, diabetes duration, history of CVD, history of diabetes complications, previous OHA use, and follow-up time); Model 3, fully adjusted as Model 2 plus pre-index HbAlc, pre-index BMI and weight $B M I$ body mass index, $H b A 1 c$ glycosylated hemoglobin, $I D$ insulin detemir, $I G$ insulin glargine, $N P H$ neutral protamine Hagedorn, $O H A$ oral hypoglycemic agent, $P M$ premixed insulin, $S D$ standard deviation

showed that patients treated with ID and PM required 59\% and 25\% higher doses, $p<0.0001$ (Table 6). The required insulin doses did not differ significantly between IG and NPH.
Hypoglycemia requiring admission to a hospital occurred infrequently. The total number of patients experiencing severe hypoglycemia was 26, with the largest 
Table 6 Adjusted comparison in dose of IG, ID, PM vs. NPH

\begin{tabular}{|c|c|c|c|c|c|c|c|c|c|}
\hline \multirow{2}{*}{$\begin{array}{l}\text { Daily insulin dose } \\
\text { (units/day kg) }\end{array}$} & \multicolumn{3}{|c|}{ Model 1, $n=3,591$} & \multicolumn{3}{|c|}{ Model 2, $n=3,350$} & \multicolumn{3}{|c|}{ Model 3, $n=1,193$} \\
\hline & Change & SD & $\overline{p \text { value }}$ & Change & SD & $p$ value & Change & SD & $p$ value \\
\hline IG vs. NPH & 1.1 & 0.03 & 0.056 & 1.0 & 0.03 & 0.25 & 1.0 & 0.052 & 0.77 \\
\hline ID vs. $\mathrm{NPH}$ & 1.4 & 0.04 & $<0.0001$ & 1.0 & 0.04 & $<0.0001$ & 1.6 & 0.069 & $<0.0001$ \\
\hline PM vs. NPH & 1.2 & 0.02 & $<0.0001$ & 1.2 & 0.02 & $<0.0001$ & 1.2 & 0.036 & $<0.0001$ \\
\hline
\end{tabular}

Multivariate analysis using generalized linear modeling. Model 1, unadjusted; Model 2, adjusted for covariates with few missing values (age, gender, level of income, diabetes duration, history of CVD, history of diabetes complications, previous OHA use, and follow-up time); Model 3, fully adjusted as Model 2 plus pre-index HbA1c, pre-index BMI and weight $B M I$ body mass index, HbAlc glycosylated hemoglobin, $I D$ insulin detemir, $I G$ insulin glargine, NPH neutral protamine Hagedorn, $O H A$ oral hypoglycemic agent, $P M$ premixed insulin, $S D$ standard deviation

Table 7 Insulin doses

\begin{tabular}{lllllc}
\hline & NPH & IG & ID & PM & $p$ value \\
\hline Insulin only & & & & & \\
Dose (U/day) & $28.1(16.0)$ & $28.7(19.3)$ & $34.2(19.2)$ & $39.8(21.7)$ & $<0.001$ \\
Total insulin dose (U/day) & $43.7(26.9)$ & $46.5(32.2)$ & $50.1(24.5)$ & $41.1(22.3)$ & 0.0186 \\
Adjusted dose (U/day $\times$ kg weight) & $0.34(0.17)$ & $0.34(0.14)$ & $0.42(0.21)$ & $0.52(0.27)$ & $<0.001$ \\
Adjusted total insulin dose (U/day $\times$ kg weight) & $0.55(0.30)$ & $0.57(0.28)$ & $0.69(0.32)$ & $0.53(0.28)$ & 0.0146 \\
Insulin and OHA combination & & & & & \\
Dose (U/day) & $29.9(17.9)$ & $30.2(17.0)$ & $42.1(29.0)$ & $41.4(24.7)$ & $<0.001$ \\
Total insulin dose $(\mathrm{U} /$ day) & $34.5(23.4)$ & $34.8(22.3)$ & $51.2(35.7)$ & $42.4(26.5)$ & $<0.001$ \\
Adjusted dose $(\mathrm{U} /$ day $\times$ kg weight $)$ & $0.33(0.18)$ & $0.33(0.16)$ & $0.45(0.31)$ & $0.47(0.24)$ & $<0.001$ \\
Adjusted total insulin dose $(\mathrm{U} /$ day $\times$ kg weight $)$ & $0.38(0.25)$ & $0.39(0.23)$ & $0.56(0.38)$ & $0.47(0.25)$ & $<0.001$ \\
\hline
\end{tabular}

$I D$ insulin detemir, $I G$ insulin glargine, $N P H$ neutral protamine Hagedorn, $O H A$ oral hypoglycemic agent, $P M$ premixed insulin, $U$ insulin units

numbers in patients treated with NPH $(n=7$ patients) and PM $(n=15$; overall $p=0.023)$.

\section{DISCUSSION}

This observational study provides information on clinical effects of different types of insulin in a real-world setting. The included patients demonstrated an HbA1c at baseline of $67-68 \mathrm{mmol} / \mathrm{mol}$ despite an average use of more than one type of OHA, corresponding to a stage of disease when insulin initiation is recommended [16]. The results showed significant reductions in $\mathrm{HbA1c}$ for patients treated with $\mathrm{NPH}$, IG, ID or PM during 12 months of follow-up, even though the reductions were relatively modest. There was no statistically significant difference in the achieved change of HbA1c between the different types of insulin. However, the required insulin doses differed between the groups. Patients treated with ID and PM required 59\% 
and $25 \%$ higher weight-adjusted daily insulin doses to achieve similar results on HbA1c when compared with patients treated with NPH. Patients treated with PM also gained more weight, illustrated by their greater increase in BMI during the study, compared with patients treated with other types of insulin. The mean number of days of follow-up was largest in IG. The recorded number of patients experiencing a hypoglycemic event was low (only 26 patients in total), but occurred predominantly in patients treated with premixed insulin.

The achieved HbA1c changes in the present study were smaller than seen in previous observational studies [10, 12, 17] and RCTs $[5,18,19]$. The insulin doses in the present study were in general similar or only slightly lower than in these previous studies, and cannot fully explain the modest HbA1c change. However, patient characteristics at inclusion differed significantly between these studies. For example, HbA1c levels at the time of insulin initiation were considerably lower in the present study than in previous studies based on data from routine clinical care $[10,12,17]$. Interestingly, a meta-analysis including 38,803 patients from 87 RCTs found a quite strong positive relationship between baseline Hba1c and the magnitude of HbA1c change [20]. Similar relationship has been reported from observational data [12]. Thus, the relatively low HbA1c levels at baseline and possibly fear of hypoglycemia might be explanations to the modest HbA1c reductions seen in the present study.

The present results are fairly consistent with previous findings. The Treating to Target in Type 2 Diabetes (4-T) Study evaluated the efficacy of basal insulin, prandial insulin, or biphasic (premixed) insulin in insulin-naïve patients with T2D [18]. After 1 year of follow-up, PM and prandial insulin were superior to basal insulin in lowering HbA1c, but at the cost of more frequent hypoglycemic episodes and more weight gain [18]. However, at 3 years of follow-up the insulin regimens were equally effective in controlling HbA1c, but the lower rate of hypoglycemia in the basal insulin group remained [21]. A recently published propensity score-matched observational study also found basal insulin and premixed insulin to be equally effective in lowering HbA1c, but with less weight gain and a lower rate of hypoglycemia with basal insulin [17]. These studies, however, did not distinguish between different types of basal insulin. Consistent with results from metaanalyses [6, 7, 22, 23] and RCTs [5], the present study did not show significant differences in achieved HbA1c reductions between NPH, IG or ID, but with higher required doses for ID. Thus, the finding of a slightly greater HbA1c reduction with IG reported in a study based on data from a primary care register in the United Kingdom was not confirmed [12].

The difficulties of obtaining valid information on hypoglycemia through registers have been recognized in previous observational studies. In the present study, only 26 patients with hypoglycemia were reported. This could be a substantial underestimation of the total number of hypoglycemia. However, the present data support a higher frequency in patients treated with PM [18] and possibly NPH, and also RCTs demonstrating less hypoglycemic episodes in patients treated with IG and ID compared to NPH $[6,24]$.

The present study has several strengths. The data were collected from the NDR database with a currently estimated coverage of more than $90 \%$ of all patients in hospital outpatient clinics and almost $80 \%$ of all patients in primary care in Sweden, suggesting it to be highly representative of clinical practice. The observational design allows for comparisons of the effectiveness of different types of insulin in 
a real-world setting, reflecting the results in routine clinical care. Such studies constitute important complementary information to RCTs, which often show a high degree of internal validity but at the cost of less generalizability. However, the observational design and absence of randomization involves limitations including a risk of confounding by indication, as well as the limited number of patients on IG and ID, the latter in particular. Despite comprehensive covariance adjustment for relevant covariates in the present study, the possibility of residual confounding due to unknown and unmeasured covariates cannot be ruled out. Furthermore, the lack of reliable data on non-severe hypoglycemia is an important limitation of the present study. When considering benefits and risks of glucose-lowering agents, the risk of hypoglycemia and other important adverse effects must be taken into account.

\section{CONCLUSION}

NPH, IG, ID and PM were found to be effective agents in lowering HbA1c in insulin-naïve patients with T2D in routine clinical care. The different types of insulin were equally effective in lowering $\mathrm{HbA1c}$, but with greater weight gain and more hypoglycemia associated with PM, and higher dose requirements for PM and ID. The effects on weight, dose and treatment persistence support the recommendation of NPH or IG as first and second choices in this group of patients requiring initiation of insulin treatment.

\section{ACKNOWLEDGMENTS}

All named authors meet the ICMJE criteria for authorship for this manuscript, take responsibility for the integrity of the work as a whole, and have given final approval for the version to be published.

B. Eliasson participated in the design of the study, interpretation of the results and wrote the manuscript.

N. Ekström participated in the interpretation of the results and wrote the manuscript.

S. Bruce Wirta participated in the design of the study and performed all statistical analyses.

A. Odén participated in the design of the study.

M. Pirouzi Fard performed all statistical analyses.

A.M. Svensson participated in the design of the study and the statistical analyses as well as the interpretation of the results and wrote the manuscript.

The Region Västra Götaland and the Swedish Association of Local Authorities and Regions funded the National Diabetes Register (NDR). The work made by Sara Bruce Wirta was funded by Sanofi-Aventis Ab (Bromma, Sweden). The funders had no role in study design, data collection and analysis and decision to publish or preparation of the manuscript.

Conflict of interest. B. Eliasson has participated in advisory boards for Sanofi, Eli Lilly and Novo Nordisk and served as a lecturer at educational meetings arranged by these companies.

S. Bruce Wirta is an employee of IMS Health (Stockholm, Sweden).

N. Ekstrom, A. Odén, M. Pirouzi Fard and A.M. Svensson declare no conflicts of interest.

Compliance with ethics. All procedures followed were in accordance with the ethics review board at the University of Gothenburg and with the Helsinki Declaration of 1975, as revised in 2000 and 2008. All included patients 
have agreed by informed consent to be registered before inclusion.

Open Access. This article is distributed under the terms of the Creative Commons Attribution Noncommercial License which permits any noncommercial use, distribution, and reproduction in any medium, provided the original author(s) and the source are credited.

\section{REFERENCES}

1. Inzucchi SE, Bergenstal RM, Buse JB, et al. Management of hyperglycaemia in type 2 diabetes: a patient-centered approach. Position statement of the American Diabetes Association (ADA) and the European Association for the Study of Diabetes (EASD). Diabetologia. 2012;55(6):1577-96.

2. Handelsman Y, Mechanick JI, Blonde L, et al. American Association of clinical endocrinologists medical guidelines for clinical practice for developing a diabetes mellitus comprehensive care plan. Endocr Pract. 2011;17(Suppl. 2):1-53 (Practice Guideline).

3. Ryden L, Standl E, Bartnik M, et al. Guidelines on diabetes, pre-diabetes, and cardiovascular diseases: executive summary. The Task Force on Diabetes and Cardiovascular Diseases of the European Society of Cardiology (ESC) and of the European Association for the Study of Diabetes (EASD). Eur Heart J. 2007;28:88-136 (Practice Guideline).

4. Ilag LL, Kerr L, Malone JK, et al. Prandial premixed insulin analogue regimens versus basal insulin analogue regimens in the management of type 2 diabetes: an evidence-based comparison. Clin Ther. 2007;29:1254-70 (Review).

5. Rosenstock J, Davies M, Home PD, et al. A randomised, 52-week, treat-to-target trial comparing insulin detemir with insulin glargine when administered as add-on to glucose-lowering drugs in insulin-naive people with type 2 diabetes. Diabetologia. 2008;51:408-16 (Multicenter Study Randomized Controlled Trial Research Support, Non-U.S. Gov't).

6. Horvath $\mathrm{K}$, Jeitler $\mathrm{K}$, Berghold A, et al. Long-acting insulin analogues versus NPH insulin (human isophane insulin) for type 2 diabetes mellitus. Cochrane Database Syst Rev. 2007:CD005613.

7. Singh SR, Ahmad F, Lal A, et al. Efficacy and safety of insulin analogues for the management of diabetes mellitus: a meta-analysis. CMAJ. 2009;180:385-9 (Meta-Analysis Research Support, Non-U.S. Gov't).

8. Pfohl M, Dippel FW, Kostev K, et al. Different persistence on initial basal supported oral therapy in Type 2 diabetics is associated with unequal distributions of insulin treatment regimens under real-life conditions in Germany. Int $\mathrm{J}$ Clin Pharmacol Ther. 2010;48:761-6.

9. Quinzler R, Ude M, Franzmann A, et al. Treatment duration (persistence) of basal insulin supported oral therapy (BOT) in Type-2 diabetic patients: comparison of insulin glargine with NPH insulin. Int J Clin Pharmacol Ther. 2012;50:24-32 (Comparative Study Research Support, Non-U.S. Gov't).

10. Fakhoury WK, Richter H, Christensen TE. Real-life dosage and clinical efficacy of biphasic insulin preparations in patients with type 2 diabetes. Adv Ther. 2010;27:859-69 (Comparative Study Research Support, Non-U.S. Gov't).

11. Sharplin P, Gordon J, Peters JR, et al. Improved glycaemic control by switching from insulin NPH to insulin glargine: a retrospective observational study. Cardiovasc Diabetol. 2009;8:3 (Comparative Study Evaluation Studies Multicenter Study Research Support, Non-U.S. Gov't).

12. Gordon J, Pockett RD, Tetlow AP, et al. A comparison of intermediate and long-acting insulins in people with type 2 diabetes starting insulin: an observational database study. Int J Clin Pract. 2010;64:1609-18 (Comparative Study Research Support, Non-U.S. Gov't).

13. Gudbjornsdottir S, Cederholm J, Nilsson PM, et al. The National Diabetes Register in Sweden: an implementation of the St. Vincent Declaration for quality improvement in diabetes care. Diabetes Care. 2003;26:1270-6 (Research Support, Non-U.S. Gov't).

14. Wettermark B, Hammar N, Fored CM, et al. The new Swedish Prescribed Drug Registeropportunities for pharmacoepidemiological research and experience from the first six months. Pharmacoepidemiol Drug Saf. 2007;16:726-35.

15. Merlo J, Lindblad U, Pessah-Rasmussen $\mathrm{H}$, et al. Comparison of different procedures to identify probable cases of myocardial infarction and stroke in two Swedish prospective cohort studies using local and national routine registers. Eur J Epidemiol. 2000;16:235-43 (Comparative Study Research Support, Non-U.S. Gov't).

16. Inzucchi SE, Bergenstal RM, Buse JB, et al. Management of hyperglycemia in type 2 diabetes: 
a patient-centered approach: position statement of the American Diabetes Association (ADA) and the European Association for the Study of Diabetes (EASD). Diabetes Care. 2012;35:1364-79.

17. Freemantle N, Balkau B, Home PD. A propensity score matched comparison of different insulin regimens 1 year after beginning insulin in people with type 2 diabetes. Diabetes Obes Metab. 2013;15(12):1120-27.

18. Holman RR, Thorne KI, Farmer AJ, et al. Addition of biphasic, prandial, or basal insulin to oral therapy in type 2 diabetes. N Engl J Med. 2007;357:1716-30.

19. Hermansen $K$, Davies $M$, Derezinski $T$, et al. A 26-week, randomized, parallel, treat-to-target trial comparing insulin detemir with NPH insulin as add-on therapy to oral glucose-lowering drugs in insulin-naive people with type 2 diabetes. Diabetes Care. 2006;29:1269-74.

20. Giugliano D, Maiorino M, Bellastella G, et al. Relationship of baseline HbA1c, HbA1c change and $\mathrm{HbA} 1 \mathrm{c}$ target of $<7 \%$ with insulin analogues in type 2 diabetes: a meta-analysis of randomised controlled trials. Int J Clin Pract. 2011;65:602-12.

21. Holman RR, Farmer AJ, Davies MJ, et al. Three-year efficacy of complex insulin regimens in type 2 diabetes. N Engl J Med. 2009;361:1736-47.

22. Rosenstock J, Dailey G, Massi-Benedetti M, et al. Reduced hypoglycemia risk with insulin glargine: a meta-analysis comparing insulin glargine with human NPH insulin in type 2 diabetes. Diabetes Care. 2005;28:950-5.

23. Swinnen SG, Simon AC, Holleman F, et al. Insulin detemir versus insulin glargine for type 2 diabetes mellitus. Cochrane Database Syst Rev. 2011:CD006383.

24. Home PD, Fritsche A, Schinzel S, et al. Metaanalysis of individual patient data to assess the risk of hypoglycaemia in people with type 2 diabetes using NPH insulin or insulin glargine. Diabetes Obes Metab. 2010;12:772-9. 